\title{
Effects of Polymer Confinement in AAO Nanocavities on the Microstructure and Properties of Polymer Materials
}

2 JAIME MARTIN ${ }^{*, \dagger}$, REBECA HERNÁNDEZ 3 and CARMEN MIJANGOS ${ }^{\ddagger}$

${ }_{4}{ }^{*}$ Centre for Plastic Electronics and Department of Materials,

5 Imperial College London, Exhibition Road, London, SW7 2AZ, UK

$6{ }^{\dagger}$ POLYMAT, University of the Basque Country

7 UPV/EHU Avenida de Tolosa 72

820018 Donostia-San Sebasti $\mu$, Spain

9 łnstituto de Ciencia y Tecnología de Polímeros (ICTP-CSIC),

10 c/ Juan de la Cierva, 328006 Madrid, Spain

\subsection{Introduction}

Nanotechnologies require the combination of materials in which at least one dimension is nanoscopic, with specific chemical functions and easy nanofabrication processes. Among the range of materials available for nanotechnology applications, polymers are attracting a great deal of attention due to their customizable chemical and 
physical properties and the fact that polymers can be easily processed at the nanoscale. Hence, the field of polymer nanotechnology is currently experiencing a rapid development. ${ }^{1-6}$

The replication of anodic aluminum oxide (AAO) templates is stablishing itself as a major approach for the fabrication of polymer nanostructures, thanks to its large versatility, low cost, and high yield. After the polymer material is subject to such a nanofabrication process, the physical properties and behavior of the resulting polymer nanostructures, namely, crystallization, molecular dynamics and many others, are altered with respect to the properties of the bulk polymer counterpart. This is due to the so-called "confinement effects" which arise as a consequence of the low dimensionality of the polymer material confined in the nanoporous AAO templates. ${ }^{7-11}$ Thus, "confinement effects" become a new tool to modulate the properties of polymer materials, thereby produce nanostructures with tailored functions and morphologies, which are more and more demanded for application as biosensors, scaffolds for cell adhesion, hydrophobic surfaces, and others. ${ }^{2}$

In recent years, there have been hundreds of reports demonstrating the feasibility of AAO templates to produce polymer nanostructures in a variety of polymers. For the fabrication of most of these nanostructures, a pre-synthesized polymer is infiltrated inside the pores either in form of melt or solution. A recently published review article summarizes the strategies developed to produce polymer nanostructures using AAO templates as well as a selection of the most relevant morphologies achieved, such as nanofibers, nanotubes and nanospheres, etc. made of polystyrene (PS), poly (methyl methacrylate) (PMMA), poly (vinylidenfluoride) (PVDF), and many other polymers and polymer-based composites. ${ }^{2}$ Moreover, that review gives detailed information concerning the influence of the confinement on some structural and dynamical behavior of polymers, such as the confirmation of amorphous chains, the glass transition phenomenon and chain dynamics, as well as on the material's properties - mechanical properties, transport properties, ferroelectric properties, thermal properties, rheological properties, etc. A brief section devoted to the use of AAO nanopores as nanoreactors for the 
in situ polymerization, was also reported to suggest an alternative to the polymer infiltration of pre-synthesized polymers. We encourage the readers to have a look at that paper in order to gain further knowledge on these subjects.

The search for innovative preparation methods of novel polymer structures via sophisticated infiltration methods of polymers into nanopores is currently very active. ${ }^{12-17}$ In this chapter, we show the most recent results involving polymer patterning with AAO templates aimed to develop novel polymer nanostructures. Specifically, we provide updated information in Section 9.2.1 about the employment of AAO templates as nanomoulds for the preparation of two different hierarchically nanostructured polymers: core-shell nanocylinders by a double infiltration method and polymer nanotubes obtained by layer-by-layer (LbL) assembly of polyelectrolytes inside AAO membranes.

Moreover, we compile here the recent developments that are enabling fine control of the features of the confined polymer material at different length scales, namely at molecular level and at the mesoscale. In Section 9.3.1, we address the question of how the spatial restriction impacts on the molecular architecture of polymer chains synthesized by radical polymerization inside nanopores, with special focus on the molecular weight, $\mathrm{Mw}$, and polydispersity of the synthesized polymer chains.

At a larger scale, i.e., at the mesoscale, the properties of polymer nanostructures are determined by their structural and compositional configuration. In Section 9.3.2, we review the recent advances on compositional design of polymer nanostructures produced via LbL deposition. Lastly, the impact of pore confinement on the structural development - solidification — of semicrystalline polymers is comprehensively described in Section 9.3.3.

\subsection{Polymer Nanostructures Obtained from AAO Templating}

Polymer nanostructures with modulated morphologies and properties can be easily achieved from the AAO templates assisted method. 
This conventional method allows tailoring the dimensions of a huge number of polymer and polymer-based composites and, therefore, of their specific polymer properties and applications. In effect, the fact that polymer dimensions can be adjusted is a fundamental advantage in the study of size-dependency properties. Over the last years, a growing scientific interest in exploring new strategies to find new routes to fabricate polymer nanostructures have garnered great attention for specific purposes. In this section, we display three novel line of attack/approach to get in a straightforward and/or direct manner new architectures and morphologies: (i) new morphologies named core-shell nanocylinders, (ii) combination of different nanomicro methods, AAO-LbL process, and (iii) in situ nanosynthesized polymers.

\subsubsection{AAO nanomolding}

\subsubsection{Double infiltration method (core-shell nanocylinders)}

In the literature, have been reported different methods to prepare sophisticated polymer nanostructures from AAO templates. ${ }^{13,16}$ More recently, with the objective of modifying the surface of the alumina nanopores with a polymer coating to study polymer crystallization under no influence of AAO walls, a double infiltration method from the melt was developed. ${ }^{17}$ This method allows to fabricate by means of a solution-free method (greener and easy to implement), a series of core-shell nanostructures (nanocylinders) which can be the subject of different studies. In fact, this method provides an easier and more versatile way to study the crystallization or dynamics properties of a confined polymer, in comparison to other possibilities such as the chemical functionalization of AAO pores (sylanization).

As an example of this methodology, a two-step polymer infiltration process was recently implemented to yield core-shell polycaprolactone-polystyrene (PCL-PS) nanocylinders. First, a PS nanotube inside the AAO template was generated by melt infiltration of PS in AAO templates of pore diameter of 350 and $140 \mathrm{~nm}$, at around $200^{\circ} \mathrm{C}$. Then, a second infiltration step of PCL was carried out to fill the nanotube and form the PCL core nanocylinder. 
Nanotubes and filled nanotubes thickness and diameters can be determined by scanning electron microscopy (SEM) and transmission electron microscopy (TEM). A correct infiltration of both polymers, meaning that the process resulted in no voids within the AAO templates was ascertained by fourier transform infrared spectroscopy (FTIR) which also allowed to calculate the weight average of each polymer. SEM images corresponding to polymer nanostructures obtained from infiltration within AAO templates of 250 and $140 \mathrm{~nm}$ are shown in Figs. 9.1(a)-9.1(d) and 9.1(e)-9.1(f), respectively. In Fig. 9.1(a), PS nanotubes are observed; in Fig. 9.1(b), PCL-filled PS nanotubes; Fig. 9.1(c) corresponds to the core-shell nanocylinders (PS-shell and the PCL-core); Fig. 9.1(d) (the same image colored in orange (PS) and blue (PCL), as a guide the eye); and Figs. 9.1(e) and 9.1(f) correspond to PS nanotubes and PCL-filled PS nanotubes, respectively.

To summarize, PCL nanofibers are covered by a PS shell, so it is not under the chemical influence of AAO walls. The crystallization of PCL nanofibers in the bulk has been studied and compared with the crystallization of PCL nanofibers inside AAO templates of the same size, showing a different behavior. It was suggested a surface versus homogeneous nucleation. ${ }^{17}$

\subsubsection{LbL assembly (polymer nanotubes)}

The LbL procedure consists of the alternate adsorption of a polyanion and a polycation onto charged macroscopic surfaces giving rise to inversion of the superficial charge with every adsorption step so that a number of consecutive deposited layers give rise to polymer materials structured in layers. ${ }^{18-20}$ Over the past few years, there is growing interest in coupling the LbL methodology with the employment of porous substrates as templates for the preparation of nanostructured polymer materials with hierarchic functional structures. ${ }^{21,22}$ In this way, the precise pore geometry of the template is combined with the organized layered structure of an LbL assembly. Ordered templates include micro and nanofluidic devices with well-defined geometries, polycarbonate $(\mathrm{PC})$ and $\mathrm{AAO}$ membranes. 

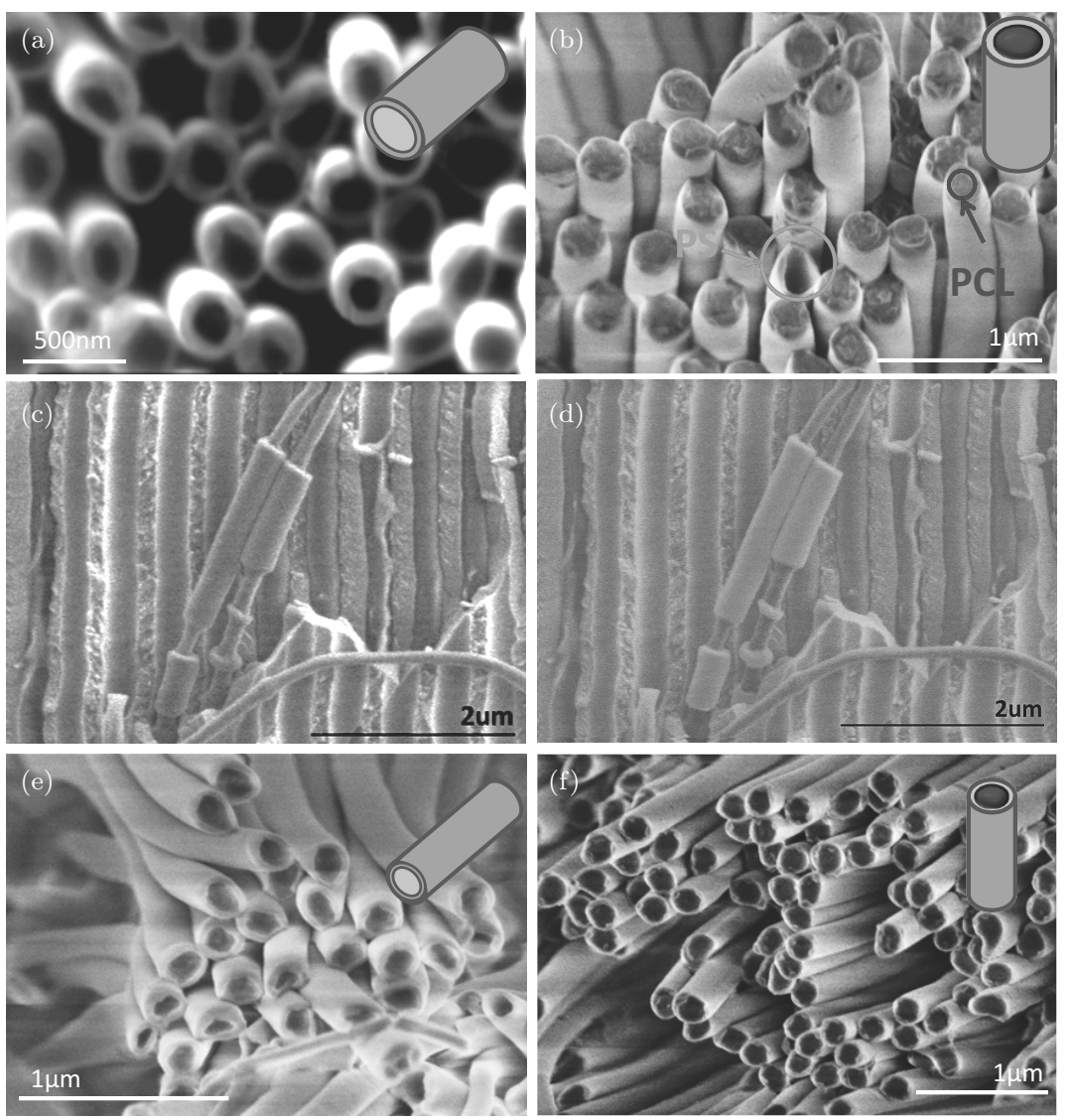

Fig. 9.1. SEM micrographs of (a) PS nanotubes after infiltration inside AAO templates with nanopores of $350 \mathrm{~nm}$ diameter, (b) PCL-PS core-shell nanostructure of $350 \mathrm{~nm}$ diameter (PCL core $220 \mathrm{~nm}$ diameter), (c) PCL-PS core-shell nanostructure of $350 \mathrm{~nm}$ diameter (PCL core $220 \mathrm{~nm}$ diameter) inside the AAO template (lateral view), (d) colored figure of (c) to guide the eye, blue for PCL core and orange for PS shell, (e) PS nanotubes after infiltration inside AAO templates with nanopores of $140 \mathrm{~nm}$ diameter, and (f) PCL-PS core-shell nanostructure of $140 \mathrm{~nm}$ diameter (PCL core $60 \mathrm{~nm}$ diameter).

Source: Reproduced with permission from Ref. [17].

A pioneering work from Caruso et al., in 2003 showed for the first 2 time the possibility to combine the LbL assembly within the AAO 3 pores. LbL assembly on AAO membranes gives rise to cylindrical 4 nanotubes with controlled external diameters in which the length, 
composition, and wall thickness can be modulated giving rise to multiwall nanotubes. ${ }^{23}$ The process of LbL inside the nanopores of 3 AAO membrane takes place experimentally by placing the membrane 4 alternatively in solutions of the polycation and the polyanion as can be observed in Fig. 9.2.

6 In order to obtain free standing multiwall nanotubes, AAO mem7 branes need to be dissolved in aqueous solutions at $\mathrm{pH}<4.5$ to $8 \mathrm{pH}>8.5$. These experimental conditions are pretty harsh toward

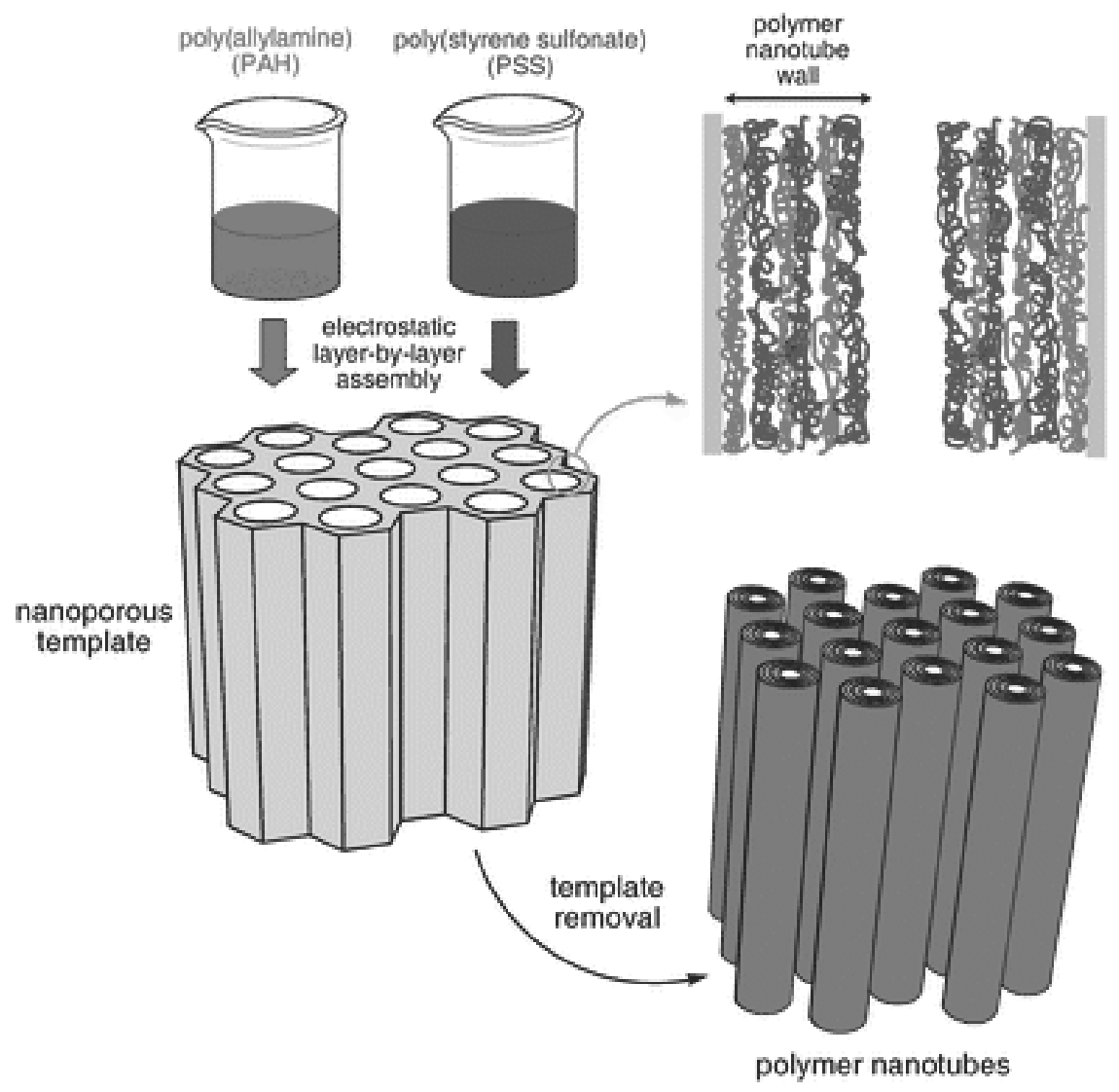

Fig. 9.2. Simplified representation of the formation of polyelectrolyte nanotubes through sequential assembly of polycations and polyanions inside the nanoporous template and the subsequent removal of the nanotemplate.

Source: Reproduced with permission from Ref. [22]. 
the polyelectrolyte assembly that holds together through ionic interactions. Alternatively, track-etched PC membranes which can be dissolved in mild solution conditions in organic solvents such as dichlorometane or dimethylformamide have also been employed for the study of the LbL assembly of different polyelectrolytes. ${ }^{24}$

Still, there is a variety of materials that have been infiltrated within the cyclindrical nanopores of AAO via the LbL assembly. One example is the deposition of protein-based nanotubes, whose chemical structure consists of polypeptidic chains with different ionizable groups for potential applications as biosensors and enzymatic bioreactors and for disease treatment and bioseparations. ${ }^{25}$ As an example of preparation, AAO membranes were alternatively immersed in an aqueous solution of a protein followed by immersion in a glutaraldehyde solution, a crosslinking agent that facilitated the stability of the protein layers after AAO membrane dissolution. The number of protein layers that make up the nanotube walls can be tuned by changing the number of alternately deposited protein glutaraldehyde cycles. ${ }^{26}$

TiO2-based composite nanotube arrays with well-defined outer diameters and lengths, largely determined by those of the AAO templates, and wall thicknesses controlled by the number of layers deposited have been prepared for applications in catalysis, chemical sensors, nanoelectrodes, and nanodevices. To that aim, AAO membranes were functionalized by first adsorbing poly(sodium 4-styrenesulfonate), PSS, onto the surfaces to form the first layer, to render it negatively charged. After washing the substrate with water, positively charged poly(diallyldimethylammonium chloride), was infiltrated to give rise to a bilayer. ${ }^{27}$

Many studies have focused on the fundamental behavior of polyelectrolyte deposition in confined geometries in order to determine differences with the process when carried out on standard, flat substrates. ${ }^{28-33}$ This topic will be developed in further detail, specifically focusing on LbL assembly on porous alumina templates, within Section 9.3.2. 


\subsubsection{AAO nanoreactor: In situ polymerization}

Due to strong requirements imposed by polymer infiltration methods in some cases, high temperature and long time, the preparation of polymer nanostructures by in situ polymerization of a precursor monomer in AAO templates has recently emerged as an alternative and efficient route to the polymer infiltration method. In polymers, chemical structure is strongly dependent on the reaction environment since the highly reactive species, catalysts, radicals and anions, and their molecular diffusions involved in the polymerization process are strongly susceptible to the reaction environment. A clear example of this influence is in the biological systems, where natural polymers produced by enzymatic catalysis are developed within regulated and well-organized molecular scale spaces, such as, channel structures layered materials or nanolevel templates. ${ }^{34}$ Therefore, the geometrical constraints of the well-defined nanochannels of a AAO template could affect the pattern of monomer insertion and chain growth process, thus offering a way to control the polymer chain structures and its macroscopic morphologies. As a consequence, of the great interest and importance on the subject, there are available in the literature quite a few studies on polymerization reactions in confined systems, such as, porous glass, porous coordination polymers, and the nanocavities of AAO templates, among others. Moreover, AAO nanochannels fulfill the requirements of a precision reactor, since the dimension can be adjusted and accuracy determined, therefore, this nanoreactor should be easily modeled.

In particular, the radical polymerization of vinyl monomers, such as, methyl methacrylate (MMA), styrene (St), and perfluoracrylate (FA) and the step-growth polymerization of a diol and a diisocyanate has been successfully achieved in the pores of AAO templates. ${ }^{35-39}$ Another illustrative example of polymerization in confinement has been reported by Tarnacka et al. upon step-growth polymerization of bisphenol-A diglycidyl ether (DGEBA) with aniline both in bulk and in AAO membranes. ${ }^{40}$ In this case, the kinetic of reaction was followed by both FTIR and Broadband Dielectric Spectroscopies. 
The first general observation is that kinetic curves do not follow sigmoidal shape that is characteristic for the autocatalytic type of chemical reactions. In a more detailed study, it was observed that (i) the polymerization was faster under confinement, compared to bulk reaction carried out at the same temperature conditions, (ii) the reaction speeds up with the degree of confinement, and finally, it was found that the initial step of the polymerization is significantly reduced or even suppressed in nanochannels. From the analysis of FTIR data with the support of Monte Carlo simulation they found that the rate of reaction is slower at the surface of the pore walls with respect to the polymerization at the core of nanochannels. Moreover, they found out that the activation barrier for the polymerization remains unchanged under confinement.

\subsubsection{In situ polymerization of $M M A$ monomer in $A A O$ templates}

The polymerization of this vinyl monomer is described in detail as an example of radical polymerization in confinement in AAO templates and the results compared to those obtained in the bulk. For the experiment, a solution of MMA and AIBN is placed on the top of a AAO template of $35 \mathrm{~nm}$ of pore diameter and $100 \mu \mathrm{m}$ of pore length, then, the absorption of the solution into the template occurs quickly as a result of the capillary force and polarity of the solution. The reaction is carried out under isothermal condition at $80^{\circ} \mathrm{C}$ and monitored as a function of time by Raman microscopy, by following the decreasing of double bond bands, or by Differential scanning calorimeter (DSC) by measuring the area of exothermic and intense peak that appears associated to polymerization reaction. In Fig. 9.3(a) is plotted the polymerization kinetics of MMA at $60^{\circ} \mathrm{C}, 70^{\circ} \mathrm{C}, 80^{\circ} \mathrm{C}$, and $90^{\circ} \mathrm{C}$ in bulk and within AAO of $35 \mathrm{~nm}$ pore diameter. As observed, the reaction within the AAO template is significantly faster compared to the bulk polymerization. It can also be observed that the initial rate of polymerization is much faster in the nanoconfined system than bulk and that the onset of the gel point occurs at slightly lower conversion. Moreover, the rate of polymerization after the gel point was 


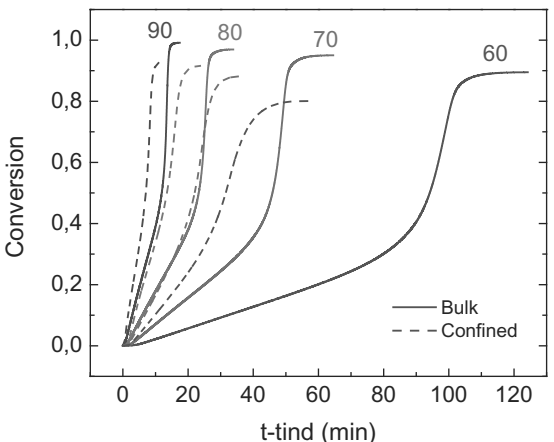

(a)

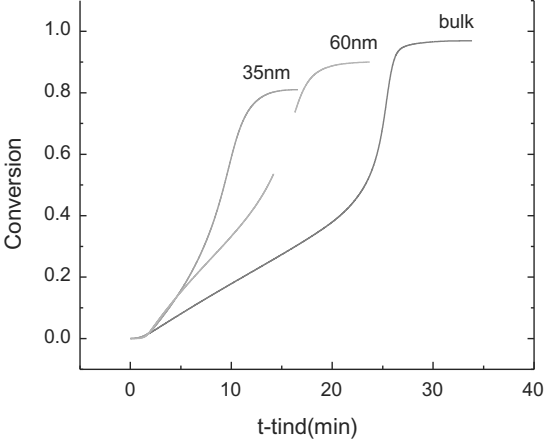

(b)

Fig. 9.3. (a) Kinetic plot of MMA polymerization in confinement at $60^{\circ} \mathrm{C}$ (blue), $70^{\circ} \mathrm{C}$ (turquoise), $80^{\circ} \mathrm{C}$ (pink), and $90^{\circ} \mathrm{C}$ (purple) (b) Evolution of conversion with time in the polymerization of MMA in bulk and within AAO templates of 35 and $60 \mathrm{~nm}$.

slower and a lower limiting conversion is reached for polymerizations under nanoconfinement. The polymerization reaction of MMA has been studied within the AAO templates at different degrees of confinements and results compared in Fig. 9.3(b). It is observed that as the degree on confinement increases (lower diameter) the reaction is faster. In an effort to parametrize the above differences, a mathematical model was implemented and is explained in Section 9.3.

\subsection{Confinement Effects on the Development of Polymeric Architecture and Microstructure}

Confinement effects of polymers within AAO templates present a strong influence on several intrinsic characteristics of polymers such as molecular weight or polydispersity and important physical properties such as self-assembly, including crystallization. In this section, we provide important information on this important area of research.

\subsubsection{Effects of confinement on the polymer architecture}

In the study of polymerization reactions in AAO templates, it is generally observed that confinement effects strongly alter the reaction 
kinetics and polymer structure, that is, molecular weight and molecular weight distribution. A general conclusion is that when a radical, controlled or step polymerization reaction is carried out in confined geometries, the polymerization kinetics and the resulting polymer structure are significantly altered. ${ }^{40-42}$ A mathematical model was implemented to study the free-radical polymerization of MMA in confinement in AAO templates and to explain the differences between the reaction kinetics and molecular weight for reactions carried out under nanoconfinement and bulk, in terms of confinement effects. Experimental data were fit using a mathematical model taking into account the following confinements effects: (i) Under confinement within the AAO walls, a faster decomposition rate of the initiator molecule leads to a faster rate of polymerization, (ii) Due to the higher glass transition temperature of polymers under nanoconfinement, the onset of diffusional limitations occurs earlier, and (iii) in the diffusion limiting region the pairwise combination of radicals also occurs faster in nanoconfinement than in bulk, due to the lower effective volume. Moreover, these confinement effects combine to give polymer of lower molecular weight and of lower dispersity. ${ }^{37}$

The experimental molecular weight distributions (MWDs) of the chains can also be simulated, as shown in Figs. 9.4(a) and 9.4(b). The lower molecular weight region consists of chains that are formed

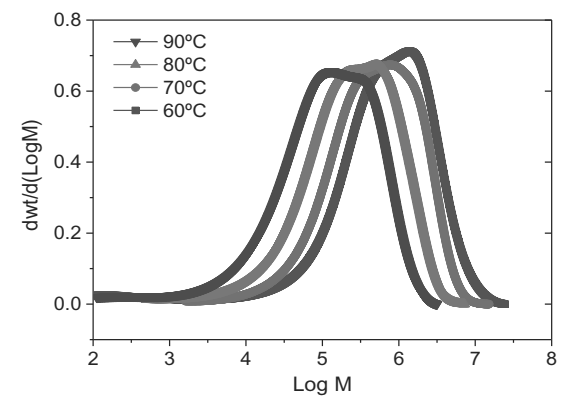

(a)

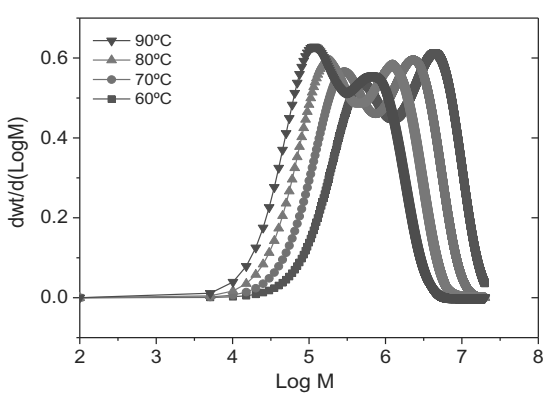

(b)

Fig. 9.4. (a) Experimental and (b) simulated molecular weight distributions of MMA polymerization in bulk at at $60^{\circ} \mathrm{C}$ (blue), $70^{\circ} \mathrm{C}$ (turquoise), $80^{\circ} \mathrm{C}$ (pink), and $90^{\circ} \mathrm{C}$ (purple). 
in the early stages of the reaction, while the higher molecular chains are generated during the second part of the reaction where, due to the low rate of termination, the kinetic chain length is increased. The simulated MWDs in Fig. 9.4(b) are in good agreement with the experimental MWD, although due to broadening of the distribution in the SEC measurement the bimodal nature of the experimental distribution is poorly defined.

\subsubsection{Effects of confinement on polymer self-assembly processes}

\subsubsection{Layer-by-layer assembly of polyelectrolytes}

The LbL assembly of polyelectrolytes within porous membranes can be employed, on the one hand, as a technique for functionalization of micro and nanoporous materials, including microfluidic devices ${ }^{43-45}$ and, on the other hand for the formation of structured materials employing the membranes as sacrificial templates. ${ }^{46,47}$ In any case, there is a need to understand the mechanism of LbL in confinement and how different experimental parameters as ionic strength, membrane characteristics or polyelectrolyte concentration influence the thickness of the resulting material.

AAO membranes provide the advantage of the control of the pore size and thickness and thus they are the nanoporous membrane of choice for fundamental studies regarding the effect of nanoconfinement in LbL assembly of different polyelectrolytes. Still, the complete understanding on the process of LbL growth on porous substrates is lagging behind. This is mainly because of the difficulty to carry out in situ measurements of film growth inside the AAO nanopores. SEM is commonly employed to visualize infiltration of polymers inside AAO membranes, however, in the case of infiltration of aqueous solutions of polyelectrolytes, the exact values of the film thickness may not be measured from SEM images as the vacuum environment in the SEM requires dried samples. Therefore, there is a need to employ in situ experimental techniques to monitor LbL polyelectrolyte growth within AAO membranes, mainly optical techniques such as interferometric reflectance spectroscopy ${ }^{48}$ 
or optical waveguide spectroscopy (OWS). ${ }^{49}$ As an example, the in situ monitoring of the LbL deposition of polyelectrolyte dendrimers, dendritic structures with ionizable groups has been carried out by means of OWS. ${ }^{32}$ These materials constitute suitable model polyelectrolytes to follow the LbL assembly within nanopores because of their well-defined peripherally charged surface and their rigid internal hydrophobic structure that results in a lower degree on interlayer penetration than that encountered for linear polyelectrolytes. ${ }^{50}$ Therefore, it is possible to determine the crossover point that defines the transition from normal assembly to assembly in nanoconfinement based on pore diameter.

Specifically, such study was carried out with fourth generation dendrimers (G4) having a theoretical diameter of $10 \mathrm{~nm}$ in a fully extended conformation and $7 \mathrm{~nm}$ in solution at high ionic strength under conditions of enhanced overall charge screening that allows an increase in the degree of dissociation. G4-polyelectrolyte LbL depositions were carried out on AAO membranes functionalized with a silane-derived molecule that provided positive surface charge. As can be observed in the SEM images corresponding to the interior and atop of a AAO membrane $(65 \mathrm{~nm})$ subjected to 10 deposition steps from $100 \mathrm{mM} \mathrm{NaCl}$ aqueous solution, the deposition takes place on the inside of the nanopore as well as on the surface of the AAO membrane (Figs. 9.5(a) and 9.5(b)). In addition, the pores in the AAO membrane were not completely filled. The thickness could be followed as a function of the number of dendrimer layers added separately for the top and the inner pore structure and the results are shown as a function of the AAO pore size in Figs. 9.5(c) and 9.5(d). The thickness of the film deposited on the surface of the AAO membrane increases linearly with the number of dendrimer layers added, similar to the trend found for the growth in planar surfaces. In addition, the growth rate is not affected by the presence of pores. However, in the case of the film infiltrated within the AAO nanopores, the film thickness linearly grows until a determined number of dendrimer layers after which a plateau in thickness is reached so that growth is inhibited beyond saturation values that decreased with pore size. That is, for $65 \mathrm{~nm}$ AAO membranes, thickness did not increase beyond 

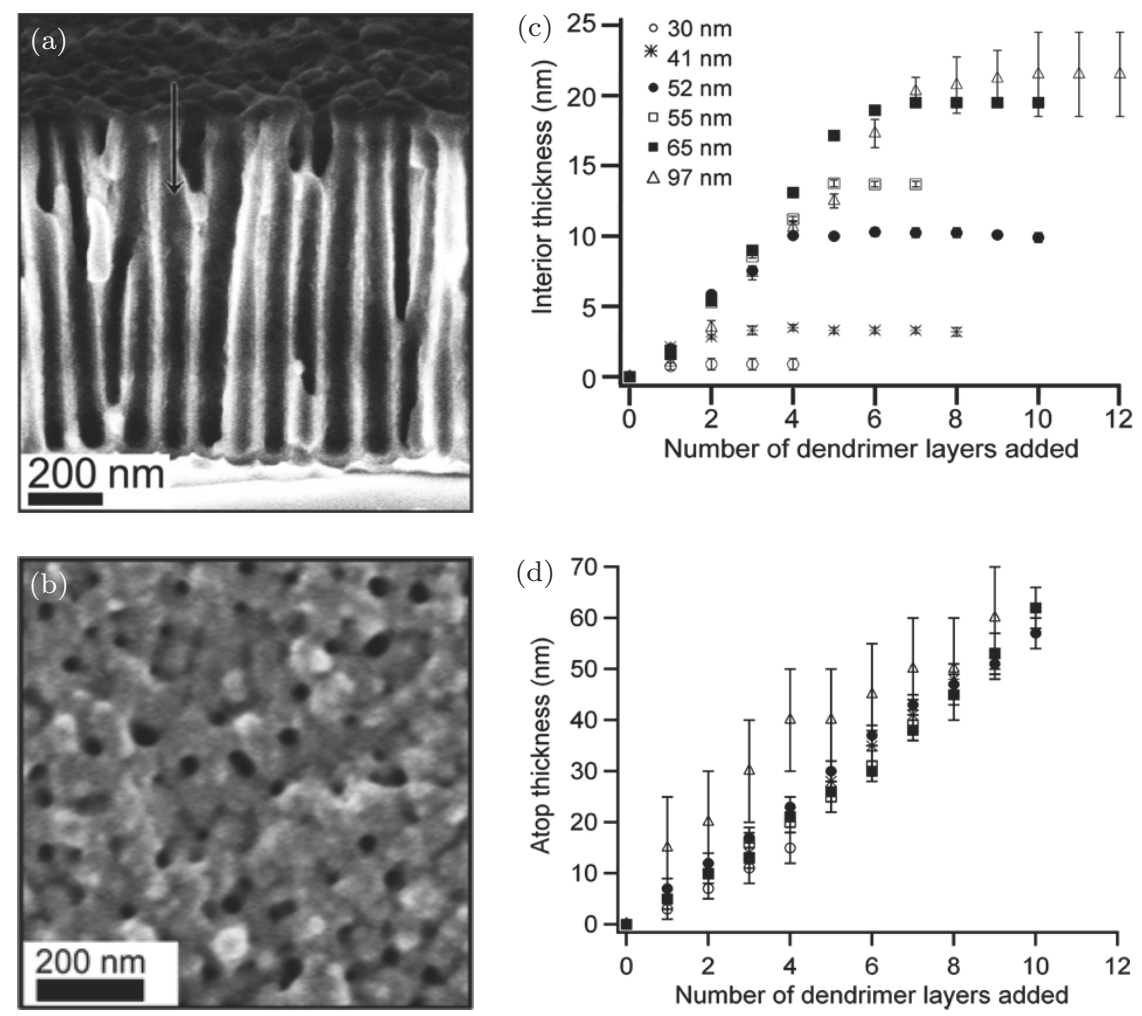

Fig. 9.5. SEM images of (a) the interior and (b) atop the AAO membrane $\left(D_{0}=65 \mathrm{~nm}\right)$ after 10 deposition steps from $100 \mathrm{mM} \mathrm{NaCl}$ aqueous solution. (c) Thicknesses of the G4-polyelectrolyte layers deposited from $100 \mathrm{mM} \mathrm{NaCl}$ aqueous solutions, within (c) and (d) atop nanoporous AAO membranes with various pore diameters.

Source: Adapted from Ref. [32].

$21.5 \mathrm{~nm}$ (6 LbL steps) while deposition within $30 \mathrm{~nm}$ pores immediately reached saturation during the first deposition step.

Rubner and coworkers studied the deposition of polymer/ 4 nanoparticle composite multilayers within AAO membranes and 5 found that the presence of superficial charge in the AAO walls 6 gives rise to electrostatic repulsion along the pore diameter which is enough to finish up the polyelectrolyte transport required for

8 LbL assembly. ${ }^{51}$ This would lead to the decrease of the level of the 
compensation charge after every layer deposition, which would result in lower thicknesses than that corresponding to the deposition on flat substrates.

Other studies have demonstrated that the growth of multilayers within the AAO membranes is independent from the molecular weight or the ionic force of the solutions and it is only dependent on the pore size at pore diameters $<250 \mathrm{~nm}^{30}$

Roy et al. demonstrated the existence of two growth regimes for polymer nanostructures within nanopores. ${ }^{33}$ The first regime is similar to the growth found in planar surfaces. The polyelectrolyte chains get close to the pore walls and get adsorbed similarly to the deposition in planar surfaces. After the deposition of a number of bilayers, the polymer chains start to interpenetrate inside the pores giving rise to the second growth regime. The second regime is slower in terms of kinetics due to the fact that diffusion is a limitant mechanism. The interpenetration of polyelectrolyte chains within AAO pores might give rise to the formation of a dense gel that can clog the pores and avoid more deposition steps. It is important to note that the polyelectrolyte chains are highly hydrated and once they are dried, the hydrated structures get shrunk resulting in a decrease of the layer thickness.

In addition to a number of experimental studies about the LbL assembly on porous substrates, there are few theoretical studies for the molecular dynamic simulation of sequential adsorption of oppositely charged species carried out on charged substrates with cylindrical pores of different sizes. In the case of modeling of the assembly of nanoparticles, similarly to experimental results, the initial increase in the film thickness is followed by a saturation regime. This was attributed to the narrowing of the pore that inhibits penetration of nanoparticles due to the electrostatic repulsion along the pore axis that prevent the penetration of the nanoparticles deep inside the pore.

As a final remark, a general conclusion that can be drawn from different fundamental studies carried out on LbL assembly inside $\mathrm{AAO}$ nanopores is that the polyelectrolyte deposition in confinement is much more sensitive to the nature of the polyelectrolytes and the 
ionic parameters compared to the deposition that takes place on planar surfaces. In many cases, the solution ionic strength corresponding to the infiltrated aqueous solutions is much greater than that required for experiments on flat surfaces in order to efficiently infiltrate the polyelectrolytes within the nanopores. In addition, the final thickness resulting from the assembly of polyelectrolytes is significantly lower in confined geometries than in standard, flat substrates.

\subsubsection{Polymer crystallization}

Crystallization can be considered as the most common self-assembly process in nature. ${ }^{52}$ Polymers typically crystallize as lamellar crystals in which individual chains assemble normal to the surfaces of lamellae adopting helical conformations. ${ }^{53,54}$ The thickness of lamellae is typically around $10 \mathrm{~nm}^{55}$ and develops approximately in the polymer chain direction. Along the in-plane directions, in contrast, the lamellae can grow up to micrometers (even milimeters). On a next level, crystalline lamellae commonly pile up separated by disordered amorphous regions, ${ }^{56}$ which are comprised of entangled chain segments, chain-ends, chain branches, chemical defects, etc. The set of lamellae tend, in turn, to organize in larger polycrystalline assemblies; for example, densely branched spherulites with sizes that can exceed milimeters ${ }^{52,55}$ are generally achieved when bulk semicrystalline polymers are cooled down from the melt.

While the thickness of lamellae is comparable to the pore diameter of AAO templates, their lateral dimensions - and thus, the size of the spherulites - surpass by far the pore diameter values. Therefore, when a polymer melt is confined in AAO nanopores, its natural crystallization process is altered. As a matter of fact, the lateral size of the pores (defined by the diameter) conflicts with the characteristic length scales of both processes the crystallization is composed of, i.e., the crystal nucleation process and the crystal-grow process, which result in drastic changes in the crystallization kinetics and the microstructure (e.g., texture,) of the confined polymer as compared to the same material with no space limitation. 


\section{(a) Crystallization Kinetics}

Crystallization kinetic studies are typically accomplished monitoring the isothermal solidification of the polymer melt, such as a thermal profile yields a material with a more uniform inner structure than that of polymers crystallized under a constant cooling rate. Moreover, the evolution of the degree of crystallinity with time under isothermal conditions can be modeled by relatively simple mathematical models, the Avrami model ${ }^{55}$ being the most frequently employed one. In the Avrami model, the advance of the crystallinity with time, i.e., the crystallization kinetics, can be expressed as:

$$
V_{c}(t)=1-\exp \left(-K t^{n}\right),
$$

where $V_{c}(t)$ is the crystallinity in terms of the volume fraction of crystals (or supracrystalline structures) that develop within a specific time $t ; K$ is a rate constant of the overall crystallization process, and $n$ is the Avrami exponent. It is clear from Eq. (9.1) that the parameters $n$ and $K$ govern the crystallization kinetics.

\section{(b) The Avrami exponent, $n$}

The Avrami exponent, $n$, relates to the time dependency of the crystallization process; thereby, provides insights about every aspect of the crystallization process that depends on time. Consequently, $n$ can be, in turn, interpreted as

$$
n=n_{n}+n_{g},
$$

where $n_{n}$ and $n_{g}$ are the contributions to $n$ resulting from how the nucleation process and crystal growth process depend on time, respectively. Typically, $n_{n}$ ranges between zero to one. For example, in purely instantaneous nucleation processes where the large majority of nuclei develop at the same time, such as in the athermal heterogeneous nucleation occurring in bulk polymers, $n_{n}=0$. Conversely, $n_{n}=1$ when nuclei are developed at constant rate, like it is frequently assumed in purely homogenous nucleation processes. 
On the other hand, $n_{g}$ informs about the dimensionality of the growth of crystals when a constant growth rate is assumed, and adopts the values one, two or three when crystals (or supracrystalline structures) develop in one, two or three spatial dimensions, respectively.

Bulk polymers typically crystallize via heterogeneous nucleation mechanism $\left(n_{n}=0\right)$ followed by a $2 \mathrm{D}$ or $3 \mathrm{D}$ spherulitic growth of crystals $\left(n_{g}=2\right.$ or 3$) .{ }^{55}$ Thus, $n\left(n=n_{n}+n_{g}\right)$ acquires values of two or three (or even higher) that result in the typical sigmoidal curves for the advance of the crystallinity $\left(V_{c}(t)\right)$ with time that are commonly observed in bulk polymer crystallization. ${ }^{55}$ In contrast, the value of $n$ drops in crystallizations developed within pores as will be described in what follows giving rise to changes of the functional form of $V_{c}(t) .{ }^{57-60}$

The main reason for the reduction of the value of $n$ in crystallizations within pores is that $n_{g}$ decreases strongly because of the restricted volume available for crystals to grow. Note that the maximum theoretical dimensionality for the growth of crystals in (highly confining) cylindrical pores is one, which is just achieved when the crystals can freely propagate straight on the pore. ${ }^{61,62}$ When the growth of crystals along the pore is impeded, $n_{g}<1$ can even be achieved. The reduction of $n_{g}$ is in general large enough to overcome the typical increase of $n_{n}$ induced by the transition from heterogeneous nucleation mechanism in bulky polymers $\left(n_{n}=0\right)$ to homogeneous nucleation mechanism that is frequently active in confined systems (where $n_{n}=1$, as will be described in detail below). ${ }^{59,63-69}$ Moreover, the dominance of the nucleation process over the crystal growth process in crystallization of confined polymers also contributes to lowering $n$, as $n_{n}$ dominates against $n_{g}$, and $n_{n}$ ranges solely between zero and one.

Michell et al. studied the crystallization of poly(ethylene oxide) (PEO) inside AAO pores of 60 and $35 \mathrm{~nm}$ in diameter. As shown in Fig. 9.6(a), $n$ in these crystallizations were of close to one (between 0.7 and 1.4), ${ }^{57}$ which was rationalized in terms of a complete suppression of the crystal growth due to the limited space $\left(n_{g}=0\right)$ 


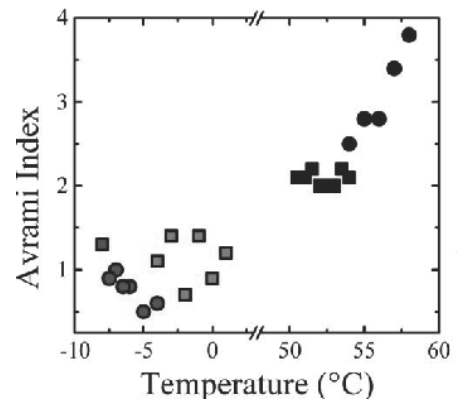

(a)

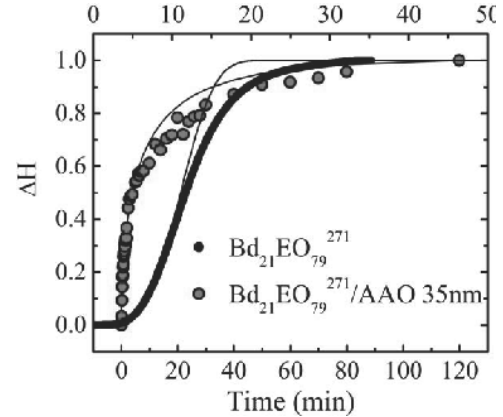

(b)

Fig. 9.6. (a) Avrami index versus $T_{c}$ for bulk PEO (black squares), PEO confined in pores of $60 \mathrm{~nm}$ (red squares), PEO confined in pores of $35 \mathrm{~nm}$ (blue squares), bulk $\mathrm{P}(\mathrm{Bd}-\mathrm{b}-\mathrm{EO})$ copolymer (black circles), and $\mathrm{P}(\mathrm{Bd}-\mathrm{b}-\mathrm{EO})$ copolymer confined in pores of $35 \mathrm{~nm}$ (blue circles). (b) Advance of crystallinity (expressed as the relative melting enthalpy values, $\Delta \mathrm{H}$ ) with time for bulk PEO-b-PB (black circles, crystallization temperature $\left(T_{c}\right)$ was $58^{\circ} \mathrm{C}$ ), and within $35 \mathrm{~nm}$ AAO templates $\left(T_{c}=-4^{\circ} \mathrm{C}\right)$. The solid lines correspond to fits to the Avrami equation. The top $x$-axis corresponds to the bulk materials while the bottom $x$-axis corresponds to that of the confined material.

Source: Extracted from Ref. [58].

and homogeneous nucleation mechanism $\left(n_{n}=1\right)$. In further studies, even lower $n$ values $(0.5-1)$ were reported by Michell et al. for $\mathrm{PEO}$ domains in a poly(ethylene oxide-b-butadiene) (PEO-b-PB) diblock copolymer ${ }^{59}$ (Fig. 9.6(a)). These crystallizations exhibited nearly-first-order-kinetics and, subsequently, an exponential trend for the crystallinity with time (Fig. 9.6(b)). Similar arguments were employed by Maiz et al. to explain the reduction of the $n$ values (ranging from 1.3 to 2.1 ) in the crystallization kinetics of polyethylene (PE) confined in AAO nanopores. ${ }^{64}$

Note that in all these studies, $n_{g}$ is the main contribution to $n$ and, unlike in typical crystallizations of bulk polymers, acquires a non-zero value. The rationale for the non-zero value of $n_{g}$ is that, under confinement, a transition frequently occurs from instantaneous, heterogeneous nucleation of neat bulk polymers to a heterogeneity-free, homogeneous nucleation process, which is timedependent. ${ }^{57-59,61,63-77}$ Bulk polymers typically crystallize via a 
1

heterogeneous nucleation mechanism originated on heterogeneities (impurities) that are present in the melt. The typical density of impurities able to cause the nucleation of common thermoplastics, such as polypropylene (PP) and poly(vinyl difluoride) (PVDF), lies in the range of $10^{6}-10^{7} \mathrm{~cm}^{-3} .{ }^{78}$ This means that, in average, there is one heterogeneity every $10^{5}-10^{6} \mu \mathrm{m}^{3}$ of crystallizable material. However, the volume of a crystallizing polymer domain in a pore with a diameter of $35 \mathrm{~nm}$ and a depth of $100 \mu \mathrm{m}$ is of $\sim 10^{-1} \mu \mathrm{m}^{3}$. Thus, the number of crystallizing domains containing heterogeneities is insignificant and, hence, bulk-like heterogeneous nucleation mechanism is residual in these samples. The confined polymer nucleates, thus, through a different, heterogeneity-free mechanism, such as the homogeneous nucleation mechanism.

Interestingly, Maiz et al. found also first-order crystallization kinetics ( $n=1.1$ and 1.2) in the isothermal crystallization of PEO nanotubes with an outer diameter of $400 \mathrm{~nm}$ and a tube-wall of $35 \mathrm{~nm} ;{ }^{71}$ however, they observed also a significantly lower value of the apparent activation energy of the crystallization of the nanotubes compared to that of the bulk PEO, which suggests that their nanotubes were not crystallizing from homogeneous nucleations. Therefore, the first-order-kinetics in this system was interpreted as arising from the heterogeneous nucleation of crystals at the pore walls of the AAO templates, followed by the 1D growth of crystals.

Lastly, it is worth mentioning that pores exceeding $100 \mathrm{~nm}$ in diameter seem to have little impact on the crystallization kinetics of polymer melts. $63,76,77$

\section{(c) The crystallization rate constant, $K$}

$K$ being the rate constant for the overall crystallization process (see Eq. (9.1)), it is influenced by the nucleation rate, the number of nuclei, and the crystal growth rate. Among these factors, the major differences between confined and non-confined crystallizations are found in the number of nuclei. If, as it has been described above, the number of heterogeneities in bulk polymers is typically of $10^{6}-10^{7} \mathrm{~cm}^{-3}$, in average, a crystalline nucleus is developed every $10^{5}-10^{6} \mu \mathrm{m}^{3}$. However, the volume of the crystallizable domains, i.e., 
that of the nanopores, lies in the range of $\sim 10^{-1} \mu \mathrm{m}^{3}$. Thus, even in the unlikely case in which just a single nucleation center is developed inside each pore, the total density of nuclei in the polymer/AAO ensemble would be six to seven orders of magnitude higher than in typical bulk polymers (the density of pore in AAO templates is $10^{8}-10^{11}$ pores $\left./ \mathrm{cm}^{-2},{ }^{79-82}\right)$. This dramatic increase of the number of nuclei in polymers confined in AAO templates provokes a substantial increase of $K$ in these systems, as compared to bulk polymers (at least at low supercoolings). Note that, like $n, K$ is also governed by the nucleation stage in crystallizations in confinement.

Woo et $a l .{ }^{69}$ reported for the first time that the $K$ values of $\mathrm{PE}$ confined in AAO pores were five orders of magnitude higher than those of unconfined, bulk PE. Likewise, Michell et al. found an increase of two orders of magnitude in the $K$ values of PEO confined in $60 \mathrm{~nm}$ pores with respect to bulk PEO. ${ }^{57}$

Because the values of $K$ are largely governed by the early stages of the crystallization process in confined polymers, $K$ and the halfcrystallization time $\left(t_{1 / 2}\right)$ exhibit similar trends when plotted against the isothermal crystallization temperature, $T_{c}$ (once the values of $K$ are elevated to the power $n^{-1}$ ). Hence, $t_{1 / 2}$ can be also understood as a measure of the isothermal crystallization rate in confined systems and compared, thus, to $K$. As a matter of fact, Michell et al. showed the corresponding increase of the values of $t_{1 / 2}$ for the same PEO system confined in $60 \mathrm{~nm}$ pores with respect to its bulk counterpart. ${ }^{58}$ Likewise, Maiz et al. found higher $t_{1 / 2}$ values in the crystallization of PE inside pores of $35 \mathrm{~nm}$ in diameter than in bulk PE. ${ }^{64}$

\section{(d) Crystal orientation (texture)}

One of the most striking effects of pore-confinement is that high degrees of crystal orientation are induced. Furthermore, in many cases, the texture of the achieved nanostructure can be selected via suitable selection of the pore size $\mathrm{e}^{72,83}$ and the presence or absence of a bulk polymer reservoir ${ }^{68,84,85}$ at the surface of the AAO template. It should be pointed out, though, that here we mainly focus on polymer nanostructures crystallized in the absence of such bulk reservoir. 
The general trend in the crystallization of polymers confined in the straight cylindrical nanopores of AAO templates is that crystals propagate laying the polymer chains normal to the long axis of the nanopores. ${ }^{61,64,65,73,75,77,83,84}$ The rationale for this orientation is that polymeric chain-folded-lamellae can just grow along lateral directions, i.e., directions that are perpendicular to chains. Hence, the orientation of chain normal to the long axis of the pores allows the lamellae to propagate straight on the pores. Using Miller index notation, the crystals having $\langle h k l\rangle$ directions with a cero $l$ index are the ones growing preferentially along the pore axis direction, as the $l$ index (i.e., the $c$-axis of the crystal lattice) is approximately parallel to the chain direction in most of the polymer unit cells. Note, therefore, that, whereas the nucleation stage dominates the crystallization kinetics, the texture tends to be governed by the kinetic aspects of the crystal grow process.

A clear example of this texture has been recently found by Martín et al. in PVDF nanotubes. ${ }^{86}$ Figure 9.7 shows the WAXS patterns of bulk PVDF and PVDF nanotubes still embedded in the AAO template (Fig. 9.7(a)). In these measurements, the scattering vector, $q$, was parallel to nanopore long axis. In the $2 \theta$-range investigated, the bulk PVDF pattern showed all the characteristic reflections of $\alpha$-PVDF, namely (100), (020), (110), and (021), ${ }^{87}$ which indicates that the crystalline moieties in these samples are isotropically oriented. The pattern of the PVDF nanotubes also contains only features for $\alpha$-PVDF, however, it displayed only reflections from the (020) and (110) lattice planes. Thus, crystals with $\langle h k l\rangle$ directions with a zero $l$-index are solely detected along the nanotube long axis direction.

In order to gain further information about the texture of PVDF nanotubes, XRD pole figure measurements were accomplished for the (020), (110), and (021) reflections (Fig. 9.7(b)). In these, the $\psi$-angle, defined as the angle produced by the rotation of the sample around an axis parallel to the plane of the template surface, is plotted along the radial direction, while the $\phi$-angle, defined as the angle produced by the rotation of the sample around the long axis of the nanotubes, is plotted along the azimuthal direction (see inset in Fig. 9.7(a) for the identification of the angles). The (020) reflection centered at $\psi=0^{\circ}$ 


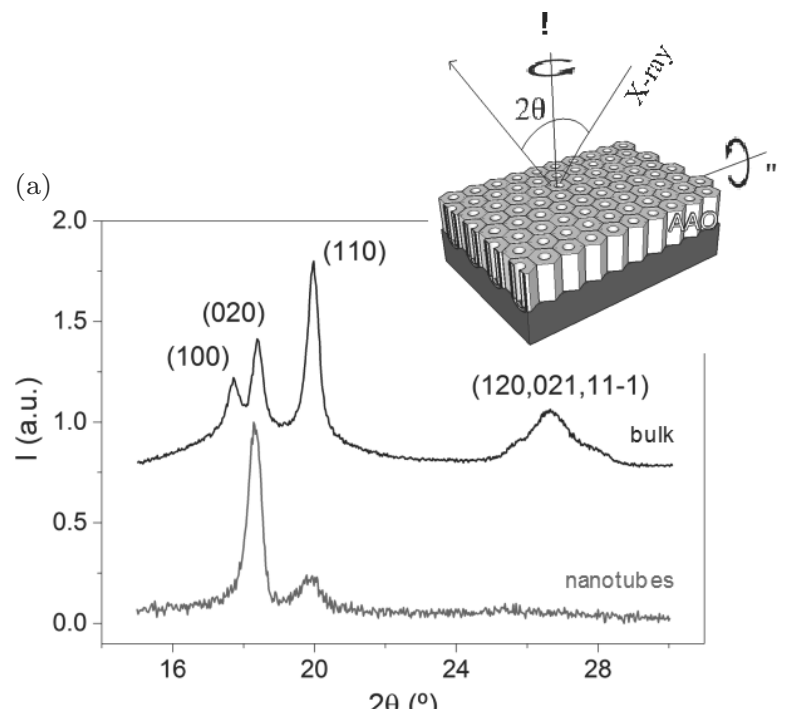

(b)

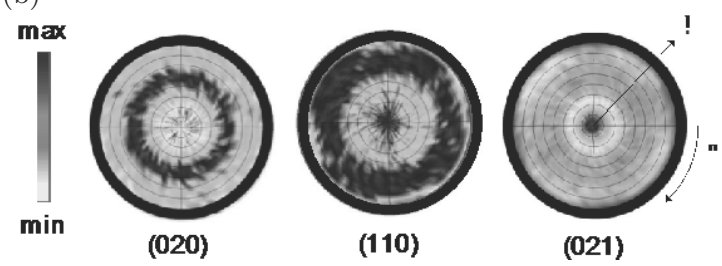

Fig. 9.7. (a) WAXS patterns of bulk PVDF and PVDF nanotubes, when $q$ is parallel to the long axis of the nanotubes, i.e., $\psi=0^{\circ}$. The geometry of the experiments as well as the relevant, scanned angles are identified the schematic (inset). (b) XRD pole figures of the (020), (110), and (021) reflections measured in PVDF nanotubes, where the radial direction corresponds to the $\psi$-angle and the azimuth corresponds to $\phi$.

Source: Adapted from Ref. [86].

revealed that the nanotubes were mainly comprised of crystals in 2 which the crystallographic $\langle 020\rangle$ direction was aligned with the nanotube long axis. These crystalline lamellae are isotropically oriented along the azimuthal $\phi$-angle, as deduced from the ring-like patterns of (110) and (210) reflections.

Similar results were obtained by Martín et al. for the semiconducting polymer poly(9,9-dioctylfluorene) (PFO) confined into $35 \mathrm{~nm}$ AAO pores. ${ }^{84}$ The 2D-WAXS pattern of this sample was 
characterized by well-defined reflection at $q=1.48 \AA^{-1}$ ( $d$-spacing of $0.425 \mathrm{~nm}$ ) that is ascribed to the stacking of (530) lattice planes of the $\alpha$-phase of PFO. A less intense reflection, corresponding to the stacking of (200) lattice planes, was also observed at $q=0.49 \AA^{-1}$. Thus, most of crystals pointed their (530) crystallographic direction parallel to the pore axis, although a small crystal fraction existed, where the $\langle 200\rangle$ direction was aligned to the pore axis. Note that, again, both crystallographic directions corresponded to the $\langle h k l\rangle$ directions with zero $l$-index and as such, the chains lay normal to the longitudinal axis of pores.

Highly texturized polymer nanostructures with a single, uniaxial orientation can be also achieved when polymer melts are crystallized inside AAO porous templates. The formation of these ultra-oriented structures seems to be associated with a low nucleation rate as well as the existence of a crystallographic direction having a significantly faster growth rate than rest of the directions. Thus, under low nucleation rate conditions, such as low cooling rates in dynamic crystallizations or high $T_{c}$ in isothermal crystallizations, some polymers crystallize aligning just the crystallographic direction with the fastest growth rate parallel to the longitudinal axis of the pores. As proposed by Huber ${ }^{88-90}$ this can be traced to a crystallization mechanism, first suggested by Bridgman, for the single crystal growth in narrow capillaries. ${ }^{91}$ Within small capillaries, the crystalline direction with higher growth rate propagate along the direction of the long axis, while other growth directions die out.

By means of 2D-WAXS, Michell et al. investigated the texture of PEO confined in pores of $20 \mathrm{~nm}$ in diameter. ${ }^{66}$ Figure 9.8 shows the 2D-WAXS patterns obtained when the X-rays traveled along the $x$-axis (a), $y$-axis (b), and $z$-axis (c) of the sample, the $z$-axis being parallel to the long axis of the nanopores, and $x$ and $y$ two directions mutually orthogonal and normal to the $z$-axis. The 2D-WAXS patterns resulting from the scattering of the PEO nanostructures in the $z-x$ and the $z-y$ planes (Fig. 9.8(a) and 9.8(b), respectively) showed the diffractions ascribed to the (120) planes located mainly along the meridian. Conversely, the 2D-WAXS pattern corresponding to the $x-y$ plane displayed ring-like signals for all the diffractions. 


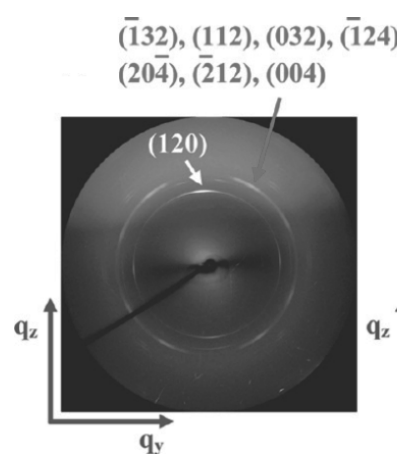

(a)

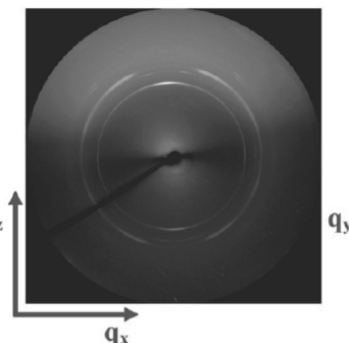

(b)

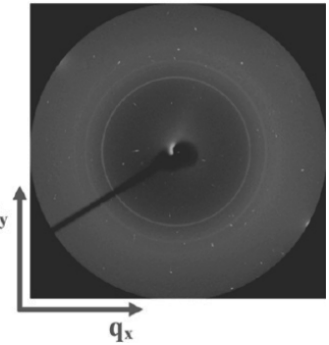

(c)

Fig. 9.8. 2D-WAXS patterns of PEO confined in $20 \mathrm{~nm}$ pores, collected for the $\mathrm{X}$-ray beam traveling along the $x$-axis (a), $y$-axis (b), and $z$-axis (c) of the sample, the $z$-axis being parallel to the long axis of the nanopores, and $x$ and $y$ two directions mutually orthogonal and normal to the $z$-axis.

On the basis of this results, they concluded that PEO crystal moieties laid their $\langle 120\rangle$ direction mainly parallel to the longitudinal axis of 3 the pores but they were randomly oriented with respect to the $x-y$ 4 plane.

Perfectly uniaxial PEO nanorods were achieved by Martín et al. employing a crystallization approach based on inducing heterogeneous crystal nuclei at one of the tips of the nanorods. ${ }^{61}$ By the inducement of efficient surface interactions between the nanoconfined polymer and a material with nucleating capacity at the pore mouths (metallic Al, see SEM images in Fig. 9.9(a) and 9.9(b)), they managed to generate heterogeneous crystal nuclei at temperatures much higher than natural nucleation temperature of the confined polymer, which was strongly depressed because of the homogeneous nucleation mechanism. Thus, when the samples were isothermally crystallized at temperature slightly below the new nucleation temperature, no nucleation centers could develop in the confined material other than those at the pore mouths. Hence, the confined PEO nucleated solely at one of the extremes of the nanopores and then the crystallites propagated unidirectionally along the pores.

These PEO nanorods exhibited perfectly uniaxial crystal orientation, where the (120) lattice plains of the monoclinic cell unit of 

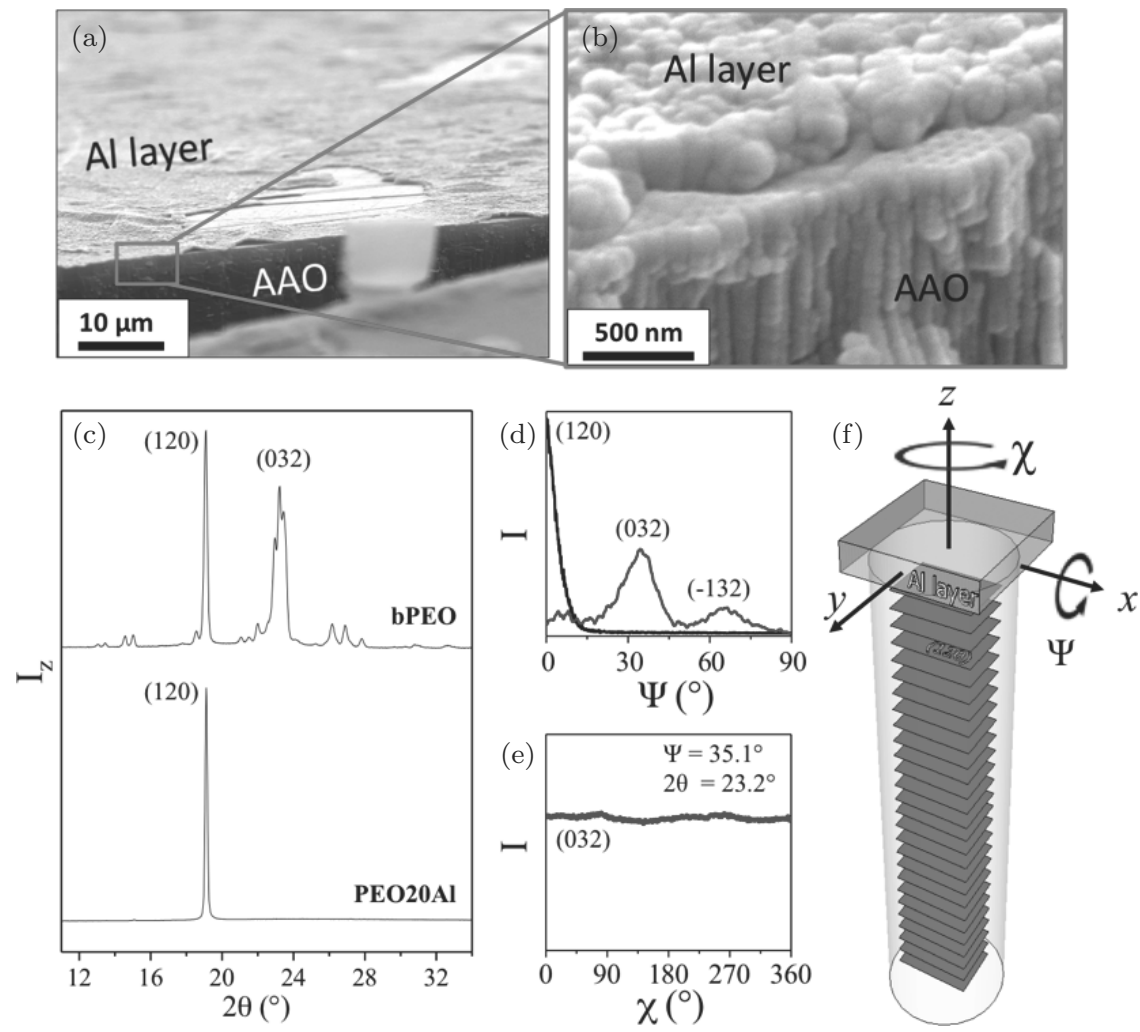

Fig. 9.9. Large view (a) and detailed view (b) SEM micrographs showing the Al layer deposited onto the AAO. (c) WAXS $\theta / 2 \theta$ scan of bulk PEO (bPEO) and confined PEO (PEO20Al) analyzed in the geometry where $q$ was parallel to the PEO nanorods ( $z$ direction). (d) WAXS intensity recorded at $2 \theta=19.1^{\circ}$ (black line) and $2 \theta=23.2^{\circ}$ (blue line) while tilting the sample around the axis defined by the intersection of template surface plane and X-ray plane of incidence ( $\Psi$ angle). (e) WAXS intensity recorded along the angle confined within the plane of the template surface for the (032) reflection. $2 \theta$ was fixed to $23.2^{\circ}$, and $\Psi$ was fixed to $35.1^{\circ}$. (e) Schematics of the crystal orientation PEO crystals in the pores (red foils represent the (120) planes of crystal lattice.

Source: Adapted from Ref. [61].

PEO were normal to pore axis (Fig. 9.9(c)). The orientation distribution of (120) planes was studied collecting WAXS intensity as tilting the sample along the $\Psi$-angle (see the schematic in Fig. 9.9(f)) 4 while setting the $2 \theta$ angle to the position of the (120) reflection. The 
WAXS signal thus acquired presented a sharp maximum centered at $\Psi=0^{\circ}$, indicating that the corresponding lattice planes lay exclusively perpendicular to the pore axis as illustrated in Fig. 9.9(f). This was further evidenced by analyzing the orientation distribution of the (032) and (-132) diffractions, which exhibited maxima just at the theoretical angles between the respective lattice planes and the (120) lattice plane (Fig. 9.9(d)).

Note that all the highly texturized structures presented here have in common that the nucleation of crystals within the pores was effectively reduced. However, there is an extreme case where the nucleation within the pores is even suppressed. This corresponds to a crystallization scenario where the polymer melt contained into the nanopores is connected through a bulk surface reservoir of material, so that the polymer contained in each pore are no longer isolated entities. In such a case, the volume of the total crystallizing material is by far larger than the average volume required for the presence of a heterogeneity. Thus, upon cooling from the melt, this polymer system behaves as a bulky system, crystallizing at the typical bulk temperatures via heterogeneous nucleation. Since the probability of finding heterogeneities in the surface reservoir is significantly higher than in the nanopores due to the larger volume of the former, the heterogeneous nuclei developed first in the reservoir and then grow isotropically through it. Some of these crystals grow in the direction of the pores and are thus able to propagate through the material contained in them. Therefore, the confined polymer crystallizes by the growth of crystals nucleated outside of the pores, i.e., no further nucleation events develop within the pores. The texture of the polymer nanostructures thus crystallized is governed solely by the crystal growth process, in such a way that, again, the "Bridgmanmechanism" 91 for the propagation of the fast-growing crystallization mode along the pore determines the crystal orientation. Hence, perfect uniaxial orientations are frequently achieved when this crystallization strategy is used. ${ }^{64,68,92,93}$

The possibility to select crystal orientations by using surface reservoirs was first discovered by Steinhart et al. ${ }^{68}$ Further on, Maiz et al. observed the same effect for PE crystallized with surface 
reservoir inside $60 \mathrm{~nm}$ in diameter AAO templates. ${ }^{64}$ Their WAXS analysis revealed a pronounced alignment of the $\langle 020\rangle$ crystallographic direction with the long axis of the pores. Interestingly, the same publication demonstrated that when PE is forced to crystallize in the absence of surface reservoir, $\langle 020\rangle,\langle 110\rangle$, and $\langle 200\rangle$ crystallographic directions align with the long axis of the nanopores. Note that, in these configurations the chains are laid perpendicular to the long axis of the nanopores, but do not correspond to a uniaxial crystal orientation.

Uniaxial orientations were also found by Martín et al. for poly(3hexylthiophene) (P3HT) inside $250 \mathrm{~nm}$ and $350 \mathrm{~nm}$ pores. These P3HT nanostructures crystallized orienting the $b$-axis of the orthorrombic P3HT crystal parallel to the long axis of nanopores. ${ }^{83,94}$ The $b$ axis is the direction along which the $\pi-\pi$ stacking occurs and, thus, it is well known to be a fastest growth direction of the crystal. Interestingly, they found that the presence of crystals with the $\langle 100\rangle$ direction parallel to pore axis increased with the reduction of the pore diameter. They hypothesized that the semirigid polymer chains adopt flat, face-on conformations at the interface with the pore walls, which would be responsible for the $\langle 100\rangle$ crystal orientation.

In line with this result, further recent works have suggested that strong confinement conditions ${ }^{72,83}$ (i.e., extremely narrow pores), high chain rigidity, ${ }^{84}$ and surface nucleation processes ${ }^{71}$ may induce crystal textures other that those with the $c$-axis of the crystal oriented normal to the pore long axis. Thus, Guan et al. reported that inside pore with diameters above $10 \mathrm{~nm}$, PEO crystals were oriented with the $\langle 120\rangle$ direction parallel to pores, whereas within $10 \mathrm{~nm}$ diameter pores crystallites grew orienting chains parallel to the long axis of the pores. ${ }^{72}$ They rationalized this phenomenon assuming that when the pore diameter is smaller than the chain's contour length, molten chains adopt anisotropic conformations to accommodate to the reduced space, which upon crystallization, template the orientation of crystals.

Similar results were reported by Maiz et al. for PEO nanotubes having $400 \mathrm{~nm}$ outer diameter and a tube-wall of $35 \mathrm{~nm} .^{71}$ Their WAXS analysis revealed that crystals with $\langle h k l\rangle$ directions with 
non-zero $l$-index where majority along the nanopore main axis. As mentioned in the previous section, they also found signs of heterogeneous nucleation in these nanotubes; thereby, they proposed a crystallization scenario where the nuclei were generated at the PEO-AAO interface and then crystals grew mainly in the radial direction of nanotubes until they reach the inner wall, where they die.

\subsection{Concluding Remarks}

Polymer 1D nanostructures derived from AAO templates, such as polymer nanowires and nanotubes, have demonstrated great potential as building blocks to produce hierarchically organized nanostructures. The recent research developments in the field are allowing fine control of the confined polymer features at the different length scales relevant for materials properties; i.e., at molecular level via in situ polymerization and, at the mesoscale, through crystallization engineering and compositional design via LbL deposition and multicomponent infiltration. Hence, further advances are expected in the future that will allow customization of properties in the 1D nanostructures as well as their integration into nanostructured devices that may lay the foundation of a future technology with applications in sensing, electronics, photonics, biomedicine, etc.

\section{References}

1. C. R. Martin, Nanomaterials: A membrane-based synthetic approach. Science, 266(5193), 1961-1966 (1994).

2. C. Mijangos, R. Hernández, and J. Martín, A review on the progress of polymer nanostructures with modulated morphologies and properties, using nanoporous AAO templates. Prog. Polym. Sci., 54-55, 148-182 (2016).

3. J. Martín et al., Tailored polymer-based nanorods and nanotubes by "template synthesis": From preparation to applications. Polymer, 53(6), 11491166 (2012).

4. J. Martín and C. Mijangos, Tailored polymer-based nanofibers and nanotubes by means of different infiltration methods into alumina nanopores. Langmuir, 25(2), 1181-1187 (2009).

5. J. Maiz, J. Sacristan, and C. Mijangos, Probing the presence and distribution of single-wall carbon nanotubes in polyvinylidene difluoride 1D nanocomposites by confocal Raman spectroscopy. Chem. Phys. Lett., 484(4-6), 290-294 (2010). 
6. A. M. Md Jani, D. Losic, and N. H. Voelcker, Nanoporous anodic aluminium oxide: Advances in surface engineering and emerging applications. Prog. Mater. Sci., 58(5), 636-704 (2013).

7. M. Steinhart et al., Polymer nanotubes by wetting of ordered porous templates. Science, 296(5575), 1997-1997 (2002).

8. D. Wu et al., Design and preparation of porous polymers. Chem. Rev., 112(7), 3959-4015 (2012).

9. H. Duran et al., Poly ( $\gamma$-benzyl-l-glutamate) peptides confined to nanoporous alumina: Pore diameter dependence of self-assembly and segmental dynamics. Macromolecules, 42(8), 2881-2885 (2009).

10. S. Ok et al., Confinement effects on chain dynamics and local chain order in entangled polymer melts. Macromolecules, 43(10), 4429-4434 (2010).

11. M. Steinhart et al., Nanotubes by template wetting: A modular assembly system. Angew. Chem. Int. Ed., 43(11), 1334-1344 (2004).

12. J.-T. Chen et al., Solvent-annealing-induced nanowetting in templates: Towards tailored polymer nanostructures. Macromol. Rapid Commun., 34(4), 348-354 (2013).

13. J.-T. Chen et al., Fabrication of polymer nanopeapods in the nanopores of anodic aluminum oxide templates using a double-solution wetting method. Macromolecules, 47(15), 5227-5235 (2014).

14. X. Feng and Z. Jin, Spontaneous formation of nanoscale polymer spheres, capsules, or rods by evaporation of polymer solutions in cylindrical alumina nanopores. Macromolecules, 42(3), 569-572 (2009).

15. S. Mei, X. Feng, and Z. Jin, Fabrication of polymer nanospheres based on rayleigh instability in capillary channels. Macromolecules, 44(6), 1615-1620 (2011).

16. H. Jo et al., Fabrication of chemically tunable, hierarchically branched polymeric nanostructures by multi-branched anodic aluminum oxide templates. Langmuir, 32(25), 6437-6444 (2016).

17. B. Sanz et al., New double-infiltration methodology to prepare PCL-PS core-shell nanocylinders inside anodic aluminum oxide templates. Langmuir, 32(31), 7860-7865 (2016).

18. G. Decher, Fuzzy nanoassemblies: Toward layered polymeric multicomposites. Science, 277(5330), 1232-1237 (1997).

19. M. Criado et al., Quantitative nanomechanical properties of multilayer films made of polysaccharides through spray assisted layer-by-layer assembly. Biomacromolecules, 18(1), 169-177 (2017).

20. J. J. Richardson, M. Björnmalm, and F. Caruso, Technology-driven layerby-layer assembly of nanofilms. Science, 348(6233) (2015).

21. F.-X. Xiao et al., Layer-by-layer assembly of versatile nanoarchitectures with diverse dimensionality: A new perspective for rational construction of multilayer assemblies. Chem. Soc. Rev., 45(11), 3088-3121 (2016).

22. O. Azzaroni and K. H. A. Lau, Layer-by-layer assemblies in nanoporous templates: Nano-organized design and applications of soft nanotechnology. Soft Matter, 7(19), 8709-8724 (2011). 
23. Z. Liang et al., Nanotubes prepared by layer-by-layer coating of porous membrane templates. Adv. Mater., 15(21), 1849-1853 (2003).

24. S. Zhang et al., Nanopapers of layer-by-layer nanotubes. J. Mater. Chem. B, 4(47), 7651-7661 (2016).

25. Q. Luo et al., Protein assembly: Versatile approaches to construct highly ordered nanostructures. Chem. Rev., 116(22), 13571-13632 (2016).

26. Y. Tian et al., Fabrication of protein nanotubes based on layer-by-layer assembly. Biomacromolecules, 7(9), 2539-2542 (2006).

27. Y. G. Guo et al., TiO2-based composite nanotube arrays prepared via layerby-layer assembly. Adv. Funct. Mater., 15(2), 196-202 (2005).

28. Y. Wang et al., Infiltration of macromolecules into nanoporous silica particles. Macromolecules, 40(21), 7594-7600 (2007).

29. A. S. Angelatos, Y. Wang, and F. Caruso, Probing the conformation of polyelectrolytes in mesoporous silica spheres. Langmuir, 24(8), 4224-4230 (2008).

30. H. Alem et al., Layer-by-layer assembly of polyelectrolytes in nanopores. Macromolecules, 40(9), 3366-3372 (2007).

31. J. P. DeRocher et al., Layer-by-layer assembly of polyelectrolytes in nanofluidic devices. Macromolecules, 43(5), 2430-2437 (2010).

32. T. D. Lazzara et al., Polyelectrolyte layer-by-layer deposition in cylindrical nanopores. ACS Nano, 4(7), 3909-3920 (2010).

33. C. J. Roy et al., Growth mechanism of confined polyelectrolyte multilayers in nanoporous templates. Langmuir, 26(5), 3350-3355 (2010).

34. K. Tajima and T. Aida, Controlled polymerizations with constrained geometries. Chem. Commun., 2000(24), 2399-2412 (2000).

35. J. M. Giussi et al., In-situ polymerization of styrene in AAO nanocavities. Polymer, 54(26), 6886-6893 (2013).

36. M. Salsamendi et al., Polymerization kinetics of a fluorinated monomer under confinement in AAO nanocavities. RSC Adv., 5(25), 19220-19228 (2015).

37. B. Sanz et al., Effect of confinement on the synthesis of PMMA in AAO templates and modeling of free radical polymerization. Macromolecules, 50(3), 811-821 (2017).

38. I. Blaszczyk Lezak, V. Desmaret, and C. Mijangos, Electrically conducting polymer nanostructures confined in anodized aluminum oxide templates (AAO). Express Polym. Lett., 10(3), 259-272 (2016).

39. B. Sanz et al., Thermally-induced softening of PNIPAm-based nanopillar arrays. Soft Matter, (2017).

40. M. Tarnacka et al., Following kinetics and dynamics of DGEBA-aniline polymerization in nanoporous native alumina oxide membranes — FTIR and dielectric studies. Polymer, 68, 253-261 (2015).

41. F. Begum, H. Zhao, and S. L. Simon, Modeling methyl methacrylate free radical polymerization: Reaction in hydrophilic nanopores. Polymer, 53(15), 3238-3244 (2012).

42. M. Tarnacka et al., Polymerization of monomeric ionic liquid confined within uniaxial alumina pores as a new way of obtaining materials with enhanced conductivity. ACS Appl. Mater. Interfaces, 8(43), 29779-29790 (2016). 
43. Q. Kang and W. Guo, 4 - Biomimetic Smart Nanopores and Nanochannels A2 - Tagliazucchi, Mario, in Chemically Modified Nanopores and Nanochannels, I. Szleifer, Ed. (William Andrew Publishing, Boston, 2017), pp. 85-102.

44. C. Cheng, A. Yaroshchuk, and M. L. Bruening, Fundamentals of selective ion transport through multilayer polyelectrolyte membranes. Langmuir, 29(6), 1885-1892 (2013).

45. A. L. Yost et al., Layer-by-layer functionalized nanotube arrays: A versatile microfluidic platform for biodetection. Microsys. Nanoeng., 1, 15037 (2015).

46. Y. Wang, A. S. Angelatos, and F. Caruso, Template synthesis of nanostructured materials via layer-by-layer assembly. Chem. Mater., 20(3), 848-858 (2008).

47. L. Zhang, A. Vidyasagar, and J. L. Lutkenhaus, Fabrication and thermal analysis of layer-by-layer micro- and nanotubes. Curr. Opin. Colloid Interface Sci., 17(2), 114-121 (2012).

48. F. S. H. Krismastuti et al., Real time monitoring of layer-by-layer polyelectrolyte deposition and bacterial enzyme detection in nanoporous anodized aluminum oxide. Anal. Chem., 87(7), 3856-3863 (2015).

49. K. H. A. Lau et al., Highly sensitive detection of processes occurring inside nanoporous anodic alumina templates: A waveguide optical study. J. Phys. Chem. B, 108(30), 10812-10818 (2004).

50. K. Sato and J.-I. Anzai, Dendrimers in layer-by-layer assemblies: Synthesis and applications. Molecules, 18(7), 8440 (2013).

51. J. Y. Kim et al., Formation of nanoparticle-containing multilayers in nanochannels via layer-by-layer assembly. Chem. Mater., 22(23), 6409-6415 (2010).

52. G. M. Whitesides and M. Boncheva, Beyond molecules: Self-assembly of mesoscopic and macroscopic components. Proc. Nat. Acad. Sci., 99(8), 47694774 (2002).

53. A. Keller, A note on single crystals in polymers: Evidence for a folded chain configuration. Philos. Mag., 2(21), 1171-1175 (1957).

54. K. H. Storks, An electron diffraction examination of some linear high polymers. J. Amer. Chem. Soc., 60(8), 1753-1761 (1938).

55. G. Strobl, The physics of polymers: Conceps for understanding their structures and behaviour, 3rd edn. Springer, (Berlin Heidelberg New York, 2007).

56. G. C. Oppenlander, Structure and properties of crystalline polymers. Sience, 159(3821), 1311-1319 (1968).

57. R. M. Michell et al., Confinement effects on polymer crystallization: From droplets to alumina nanopores. Polymer, 54(16), 4059-4077 (2013).

58. R. M. Michell et al., Confined crystallization of polymers within anodic aluminum oxide templates. J. Polym. Sci. B: Polym. Phys., 52(18), 1179-1194 (2014).

59. R. M. Michell et al., Confinement induced first order crystallization kinetics for the poly(ethylene oxide) block within a PEO-b-PB diblock copolymer 
infiltrated within alumina nano-porous template. Macromol. Symp., 337(1), 109-115 (2014).

60. R. M. Michell and A. J. Müller, Confined crystallization of polymeric materials. Prog. Polym. Sci., 54-55, 183-213 (2016).

61. J. Martín, A. Nogales, and C. Mijangos, Directional crystallization of $20 \mathrm{~nm}$ width polymer nanorods by the inducement of heterogeneous nuclei at their tips. Macromolecules, 46(18), 7415-7422 (2013).

62. Y. Ma et al., Undertanding crystal orientation in quasi-one-dimensional polymer systems. Soft Matter, 4, 540-543 (2008).

63. H. Duran et al., From heterogeneous to homogeneous nucleation of isotactic poly(propylene) confined to nanoporous alumina. Nano Lett., 11(4), 16711675 (2011).

64. J. Maiz et al., How gold nanoparticles influence crystallization of polyethylene in rigid cylindrical nanopores. Macromolecules, 46(2), 403-412 (2013).

65. J. Martín et al., Segmental dynamics of semicrystalline poly(vinylidene fluoride) nanorods. Macromolecules, 42(14), 5395-5401 (2009).

66. R. M. Michell et al., The crystallization of confined polymers and block copolymers infiltrated within alumina nanotube templates. Macromolecules, 45(3), 1517-1528 (2012).

67. K. Shin et al., Crystalline structures, melting, and crystallization of linear polyethylene in cylindrical nanopores. Macromolecules, 40(18), 6617-6623 (2007).

68. M. Steinhart et al., Coherent kinetic control over crystal orientation in macroscopic ensembles of polymer nanorods and nanotubes. Phys. Rev. Lett., 97(2), 027801 (2006).

69. E. Woo et al., From homogeneous to heterogeneous nucleation of chain molecules under nanoscopic cylindrical confinement. Phys. Rev. Lett., 98(13), 4 (2007).

70. J. L. Lutkenhaus et al., Confinement effects on crystallization and curie transitions of poly(vinylidene fluoride-co-trifluoroethylene). Macromolecules, 43(8), 3844-3850 (2010).

71. J. Maiz, J. Martin, and C. Mijangos, Confinement effects on the crystallization of poly(ethylene oxide) nanotubes. Langmuir, 28(33), 12296-12303 (2012).

72. Y. Guan et al., Manipulating crystal orientation of poly(ethylene oxide) by nanopores. ACS Macro Lett., 2(3), 181-184 (2013).

73. N. Shingne et al., Formation, morphology and internal structure of onedimensional nanostructures of the ferroelectric polymer P(VDF-TrFE). Polymer, 54(11), 2737-2744 (2013).

74. Y. Suzuki et al., Homogeneous crystallization and local dynamics of poly(ethylene oxide) (PEO) confined to nanoporous alumina. Soft Matter, 9, 2621-2628 (2013).

75. Y. Guan et al., Enhanced crystallization from the glassy state of poly(l-lactic acid) confined in anodic alumina oxide nanopores. Macromolecules, 48(8), 2526-2533 (2015). 
76. D. K. Reid et al., Crystallization and orientation of isotactic poly(propylene) in cylindrical nanopores. J. Polym. Sci. B: Polym. Phys., 52(21), 1412-1419 (2014).

77. Y. Suzuki et al., Multiple nucleation events and local dynamics of poly([varepsilon]-caprolactone) (PCL) confined to nanoporous alumina. Soft Matter, 9(38), 9189-9198 (2013).

78. S. Schneider et al., Self-nucleation and enhanced nucleation of polyvinylidene fluoride ( $\alpha$-phase). Polymer, 42(21), 8787-8798 (2001).

79. C. V. Manzano, J. Martín, and M. S. Martín-González, Ultra-narrow $12 \mathrm{~nm}$ pore diameter self-ordered anodic alumina templates. Microporous Mesoporous Mater., 184(0), 177-183 (2014).

80. J. Martín, C. V. Manzano, and M. Martín-González, In-depth study of selfordered porous alumina in the 140-400 nm pore diameter range. Microporous Mesoporous Mater., 151(0), 311-316 (2012).

81. C. Sun et al., Self-ordered anodic alumina with continuously tunable pore intervals from 410 to $530 \mathrm{~nm}$. ACS Appl. Mater. Interfaces, 2(5), 1299-1302 (2010).

82. H. Masuda and K. Fukuda, Ordered metal nanohole arrays made by a two-step replication of honeycomb structures of anodic alumina. Science, 268(5216), 1466-1468 (1995).

83. J. Martin et al., Poly(3-hexylthiophene) nanowires in porous alumina: Internal structure under confinement. Soft Matter, 10(18), 3335-3346 (2014).

84. J. Martin et al., Confinement effects on the crystalline features of poly(9,9dioctylfluorene). Eur. Polym. J., 81, 650-660 (2016).

85. M. Steinhart, Supramolecular organization of polymeric materials in nanoporous hard templates. Adv. Polym. Sci., 220, 123-187 (2008).

86. J. Martín et al., Relaxations and relaxor-ferroelectric-like response of nanotubularly confined poly(vinylidene fluoride). Chem. Mater., 29(8), 35153525 (2017).

87. A. J. Lovinger, Poly(Vinylidene Fluoride), in Developments in Crystalline Polymers - 1, D. C. Bassett, Ed. (Springer Netherlands, Dordrecht, 1982), pp. $195-273$.

88. P. Huber, Soft matter in hard confinement: Phase transition thermodynamics, structure, texture, diffusion and flow in nanoporous media. J. Phys. Condens. Matter, 27(10), 103102 (2015).

89. A. Henschel, P. Huber, and K. Knorr, Crystallization of medium-length 1-alcohols in mesoporous silicon: An x-ray diffraction study. Phys. Rev. E, 77(4), 042602 (2008).

90. A. Henschel et al., Preferred orientation of n-hexane crystallized in silicon nanochannels: A combined x-ray diffraction and sorption isotherm study. Phys. Rev. E, 79(3), 032601 (2009).

91. P. W. Bridgman, Certain physical properties of single crystals of tungsten, antimony, bismuth, tellurium, cadmium, zinc and tin. Proc. Am. Acad. Arts Sci., 60, 306-383 (1925). 
92. I. Martín-Fabiani et al., Crystallization under one-dimensional confinement in alumina nanopores of poly(trimethylene terephthalate) and its composites with single wall carbon nanotubes. ACS Appl. Mater Interfaces, 5(11), 53245329 (2013).

93. M. Steinhart et al., Curvature-directed crystallization of poly(vinylidene difluoride) in nanotube walls. Macromolecules, 36(10), 3646-3651 (2003).

94. M. M. Muñoz et al., Decrease in thermal conductivity in polymeric P3HT nanowires by size-reduction induced by crystal orientation: New approaches towards organic thermal transport engineering. Nanoscale, 6, 6858-7865 (2014). 